\title{
HEAVY-ION RESULTS FROM THE CMS EXPERIMENT*
}

\author{
BożEna BoIMSKA \\ on behalf of the CMS Collaboration \\ National Centre for Nuclear Research \\ Hoża 69, 00-681 Warszawa, Poland
}

(Received April 28, 2014)

\begin{abstract}
The Compact Muon Solenoid experiment at the LHC accelerator uses various physics observables to study a hot and dense medium created in heavy-ion collisions. Understanding of the medium properties requires conducting studies for different colliding systems; these include $\mathrm{PbPb}$ collisions but also reference $p p$ and $p \mathrm{~Pb}$ data. In this paper, an overview of some selected experimental results concerning collective effects, the jet-quenching phenomenon as well as the suppression of quarkonium states is presented. A special emphasis is put on the results obtained for recently acquired $p \mathrm{~Pb}$ data.
\end{abstract}

DOI:10.5506/APhysPolB.45.1291

PACS numbers: 25.75.- $\mathrm{q}, 13.85 .-\mathrm{t}$

\section{Introduction}

Quantum Chromodynamics (QCD) predicts that at a very high temperature of $150-190 \mathrm{MeV}$, corresponding to an energy density of about $1 \mathrm{GeV} / \mathrm{fm}^{3}$, nuclear matter undergoes a phase transition to a state of deconfined quarks and gluons. This new form of matter is called Quark-Gluon Plasma (QGP) and is studied experimentally in high-energy heavy-ion collisions.

The heavy-ion programme carried out by the Compact Muon Solenoid (CMS) experiment at the Large Hadron Collider (LHC) includes studies of lead-lead $(\mathrm{PbPb})$ collisions as well as analyses for reference systems, i.e. proton-lead $(p \mathrm{~Pb})$ and proton-proton $(p p)$ collisions. High centre-of-mass energies reached in collisions at the LHC allow the CMS experiment to study QCD in unexplored, so far, kinematic domain.

* Presented at the Cracow Epiphany Conference on the Physics at the LHC, Kraków, Poland, January 8-10, 2014. 
The CMS is a general purpose detector which comprises a number of subsystems. Its main parts are the tracking system, calorimeters and the muon system, which are covering a wide pseudorapidity ${ }^{1}$ range. The central feature of the CMS apparatus is a superconducting solenoid (3.8 T field). The highprecision tracker, consisting of silicon pixel and silicon strip detector modules, is located inside the solenoid. It measures charged particles within the pseudorapidity range $|\eta|<2.5$. The electromagnetic calorimeter (ECAL) and hadron calorimeter (HCAL) are positioned inside the solenoid as well. The high-granularity ECAL consists of lead-tungstate crystals distributed in a barrel region $(|\eta|<1.5)$ and two endcaps that extend to $|\eta|=3.0$. The HCAL barrel and endcaps are sampling calorimeters composed of brass and scintillator plates, covering $|\eta|<3$.0. In addition to the barrel and endcap detectors, there are also calorimeters in the forward region. Steel/quartzfiber hadronic forward calorimeters (HF) cover the $2.9<|\eta|<5.2$ range. The gas-ionization muon chambers, embedded in the steel return yoke outside the solenoid, measure muons for $|\eta|<2.4$. The subdetectors are complemented by a flexible two-level trigger system. The combination of a fast Level-1 (L1) trigger with a sophisticated High Level Trigger (HLT) allows for an efficient selection of the most interesting events, e.g. events with highmomentum jet, photon, (di)muon or high-multiplicity events. A detailed description of the CMS detector can be found in Ref. [1].

The exceptional capabilities of CMS enable studies of a wide range of heavy-ion physics related observables. The observables concern the bulk particle production, collective phenomena and, in particular, the so-called hard probes. As nuclei are extended objects, a value of the collision impact parameter $(b)$ - connected with the collision centrality - strongly influences particle production processes in heavy-ion collisions. Therefore, the observables are analysed in bins of the collision centrality. Centrality can be specified by giving a percentage of the total inelastic hadronic cross section, with $0 \%$ denoting the most central collisions $(b=0)$ and $100 \%$ corresponding to the most peripheral collisions. The number of participating nucleons, $N_{\text {part }}$, and the number of binary nucleon-nucleon collisions, $N_{\text {coll }}$, are used as measures of centrality as well. In order to obtain $N_{\text {part }}$ and $N_{\text {coll }}$ values, the energy measured in HF calorimeters (or event multiplicity from the silicon tracker) is compared to simulations including an initial collision geometry description based on the Glauber model [2] calculations and the response of the detectors.

The CMS heavy-ion data samples were recorded in the years 2010-2013. These include two sets of $\mathrm{PbPb}$ data at a nucleon-nucleon centre-of-mass energy $\sqrt{s_{N N}}=2.76 \mathrm{TeV}$, one comprising an integrated luminosity of $8.3 \mu \mathrm{b}^{-1}$

\footnotetext{
${ }^{1}$ Pseudorapidity $\eta$ is defined as $\eta=-\ln (\tan (\theta / 2))$, where $\theta$ is the polar angle of the particle's trajectory with respect to the anti-clockwise beam direction.
} 
(2010 PbPb Run) and another one with $150 \mu \mathrm{b}^{-1}$ (2011 PbPb Run). In addition, two samples of $p p$ reference data at the same centre-of-mass energy $(2.76 \mathrm{TeV})$ were collected in 2011 and 2013; integrated luminosities of $0.231 \mathrm{pb}^{-1}$ and $5.4 \mathrm{pb}^{-1}$, respectively. In September 2012, a test run of $p \mathrm{~Pb}$ collisions $^{2}$ at $\sqrt{s_{N N}}=5.02 \mathrm{TeV}$ resulted in the integrated luminosity of about $1 \mu \mathrm{b}^{-1}$. Finally, a high-statistics $p \mathrm{~Pb}$ data sample $\left(31 \mathrm{nb}^{-1}\right)$ was recorded in 2013.

\section{Experimental results: $\mathrm{PbPb}$ vs. $p \mathrm{~Pb}$ collisions}

A complete information on all of the CMS heavy-ion physics results can be found on the "Public Physics Results" web page [3]. This paper focuses only on some selected results concerning collective phenomena (including two-particle correlations), jet quenching and quarkonia suppression. More emphasis is put on studies of the proton-lead collisions for which final-state medium effects are expected to be largely absent, and which serve as baseline measurements for the study of $\mathrm{PbPb}$ collisions. However, initial state effects, such as the modification of parton distribution functions in the bound nucleons of lead ions (nPDFs), may contribute to the observed physics signals allowing for a characterization of such effects relative to a proton-proton reference.

\subsection{Signals of (possible) collective behaviour}

\subsubsection{Two-particle correlations}

Particle correlation studies are being extensively pursued in high-energy physics experiments as they may shed light on the underlying mechanism of particle production. Experiments at the Relativistic Heavy Ion Collider (RHIC) measured two-particle correlations using two-dimensional $\Delta \eta-\Delta \phi$ correlation functions, where $\Delta \eta$ is the difference in pseudorapidity and $\Delta \phi$ is the difference in azimuthal angle between the two particles. For gold-gold $(\mathrm{AuAu})$ collisions at $\sqrt{s_{N N}}=200 \mathrm{GeV}$, the RHIC experiments have found the so-called "ridge" effect $[4,5]$, i.e. the long-range (large $\Delta \eta$ ) near-side $(\Delta \phi \approx 0)$ two-particle correlations. At the LHC, a similar correlation structure has also been observed by the CMS experiment in lead-lead collisions at $\sqrt{s_{N N}}=2.76 \mathrm{TeV}[6,7]$, as well as, unexpectedly, in very high-multiplicity $p p$ collisions at $7 \mathrm{TeV}[8]$. In $p \mathrm{~Pb}$ collisions at $\sqrt{s_{N N}}=5.02 \mathrm{TeV}$, the first measurement of the ridge phenomenon was also performed by CMS [9].

${ }^{2}$ The collision energy for the $p \mathrm{~Pb}$ system was different than for $\mathrm{PbPb}$ and $p p$ collisions due to different rigidities of the proton and $\mathrm{Pb}$-ion. 
A new, detailed analysis [10] of two-particle correlations in $p \mathrm{~Pb}$ and $\mathrm{PbPb}$ collisions was performed in classes of track multiplicity ${ }^{3}, N_{\text {trk }}^{\text {offline }}$, following the procedure established for $p p$ interactions [8]. Particle pairs were formed by associating every trigger particle, with a transverse momentum within the $p_{\mathrm{T}}^{\text {trig }}$ interval, with all other charged particles with $p_{\mathrm{T}}$ from the $p_{\mathrm{T}}^{\text {assoc }}$ interval. The per-trigger-particle associated yield was defined as

$$
\frac{1}{N_{\text {trig }}} \frac{d^{2} N^{\text {pair }}}{d \Delta \eta d \Delta \phi}=B(0,0) \frac{S(\Delta \eta, \Delta \phi)}{B(\Delta \eta, \Delta \phi)},
$$

where $N_{\text {trig }}$ is the number of trigger particles in the event, the signal distribution, $S(\Delta \eta, \Delta \phi)$, is the per-trigger-particle yield of particle pairs from the same event

$$
S(\Delta \eta, \Delta \phi)=\frac{1}{N_{\text {trig }}} \frac{d^{2} N^{\text {same }}}{d \Delta \eta d \Delta \phi},
$$

and the background distribution, $B(\Delta \eta, \Delta \phi)$, is the per-trigger-particle yield of particle pairs from the mixed events

$$
B(\Delta \eta, \Delta \phi)=\frac{1}{N_{\text {trig }}} \frac{d^{2} N^{\mathrm{mix}}}{d \Delta \eta d \Delta \phi} .
$$

The mixed-event background distribution was constructed by pairing the trigger particles in each event with the associated particles from 10 different random events, excluding the original event. The signal and background distributions were first calculated for each event, and then averaged over all events within the track multiplicity class.

Figure 1 shows the two-dimensional (2D) two-particle correlation functions measured in $2.76 \mathrm{TeV} \mathrm{PbPb}$ and $5.02 \mathrm{TeV} p \mathrm{~Pb}$ collisions, panels (a) and (b) respectively. The correlations were calculated for pairs of charged particles with $1<p_{\mathrm{T}}^{\text {trig }}<3 \mathrm{GeV} / c$ and $1<p_{\mathrm{T}}^{\text {assoc }}<3 \mathrm{GeV} / c$, for events with the track multiplicity in the range $220 \leq N_{\text {trk }}^{\text {offline }}<260$. For both highmultiplicity systems, in addition to the correlation peak near $(\Delta \eta, \Delta \phi)=$ $(0,0)$ due to jet fragmentation, a pronounced long-range structure is seen at $\Delta \phi \approx 0$ extending over at least 4.8 units of $|\Delta \eta|$ (the near-side ridge). On the away-side $(\Delta \phi \approx \pi)$ of the correlation functions, the long-range structure is also observed. The away-side correlations contain the contribution from back-to-back jets and also the contribution from the ridge effect, as discovered by ALICE [11].

${ }^{3}$ Track multiplicity, $N_{\text {trk }}^{\text {offline }}$, is defined as the number of charged particles reconstructed in the silicon tracker and having $|\eta|<2.4$ and $p_{\mathrm{T}}>0.4 \mathrm{GeV} / c$. 

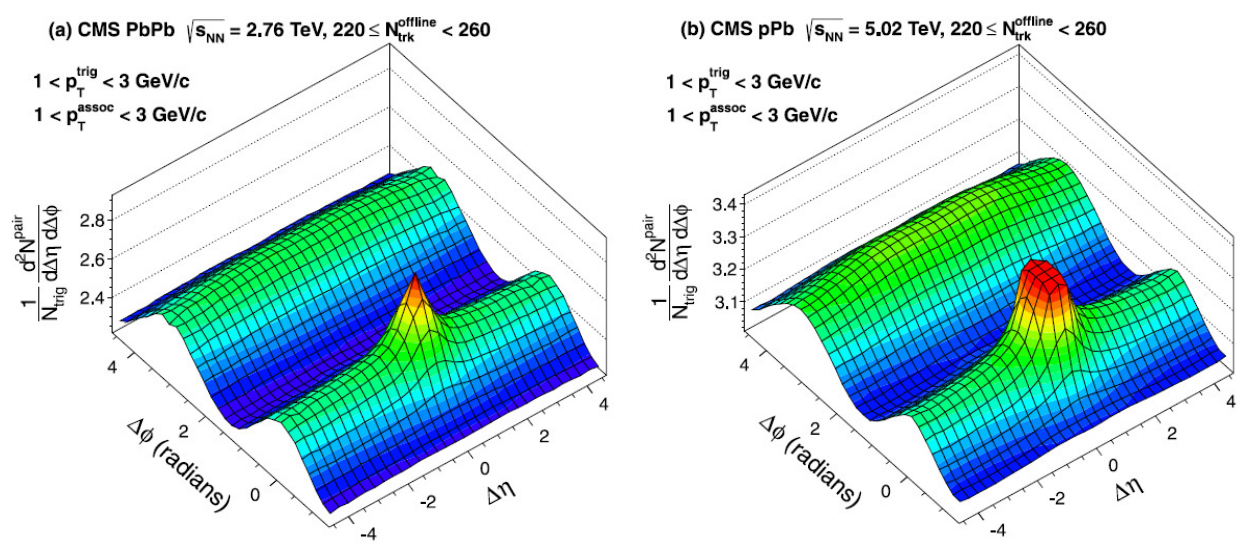

Fig. 1. Two-dimensional (2D) two-particle correlation functions for (a) $2.76 \mathrm{TeV}$ $\mathrm{PbPb}$ and (b) $5.02 \mathrm{TeV} p \mathrm{~Pb}$ collisions for pairs of charged particles with $1<p_{\mathrm{T}}^{\text {trig }}<$ $3 \mathrm{GeV} / c$ and $1<p_{\mathrm{T}}^{\text {assoc }}<3 \mathrm{GeV} / c$ within the $220 \leq N_{\text {trk }}^{\text {offline }}<260$ multiplicity bin $[10]$.

In order to investigate the observed correlations in detail and to obtain a quantitative comparison of the structure in different colliding systems, one-dimensional (1D) distributions in $\Delta \phi$ have been calculated by averaging the signal and background 2D distributions over $|\Delta \eta|>2$ (defining the long-range region). Next, the strength of the near-side long-range correlations was quantified by integrating the event-normalized associated yield over $|\Delta \phi|<1.2$. The resulting integrated yields are shown in Fig. 2 as a function of the trigger particle transverse momentum, $p_{\mathrm{T}}^{\text {trig }}$, and event multiplicity, $N_{\mathrm{trk}}^{\text {offline }}$. For high-multiplicity $\left(220 \leq N_{\mathrm{trk}}^{\mathrm{offline}}<260\right) p \mathrm{~Pb}$ and $\mathrm{PbPb}$ collisions, the near-side ridge is the most apparent in the intermediate transverse momentum range $\left(1<p_{\mathrm{T}}^{\text {trig }}<6 \mathrm{GeV} / c\right)$ - left panel of Fig. 2. As shown in the right panel, the ridge associated yield increases with the event multiplicity in both $\mathrm{PbPb}$ and $p \mathrm{~Pb}$ collisions, but also in the $p p$ system. The results indicate that the strength of the ridge effect depends on a size of the interacting system, i.e. the long-range near-side correlations are the weakest for $p p$, the middle ones for $p \mathrm{~Pb}$, and the strongest for $\mathrm{PbPb}$ collisions. However, the general characteristics for all the three systems are very similar. Does it mean that the similarities observed between these collision systems are due to the same origin of the effect?

While the ridge effect in high-energy nucleus-nucleus $(A A)$ collisions is widely accepted to be a consequence of the fluctuations of the initial geometry and the collective hydrodynamical evolution of the produced strongly interacting medium, various competing theoretical concepts have been proposed to explain the origin of this phenomenon in $p p$ and $p \mathrm{~Pb}$ collisions. 

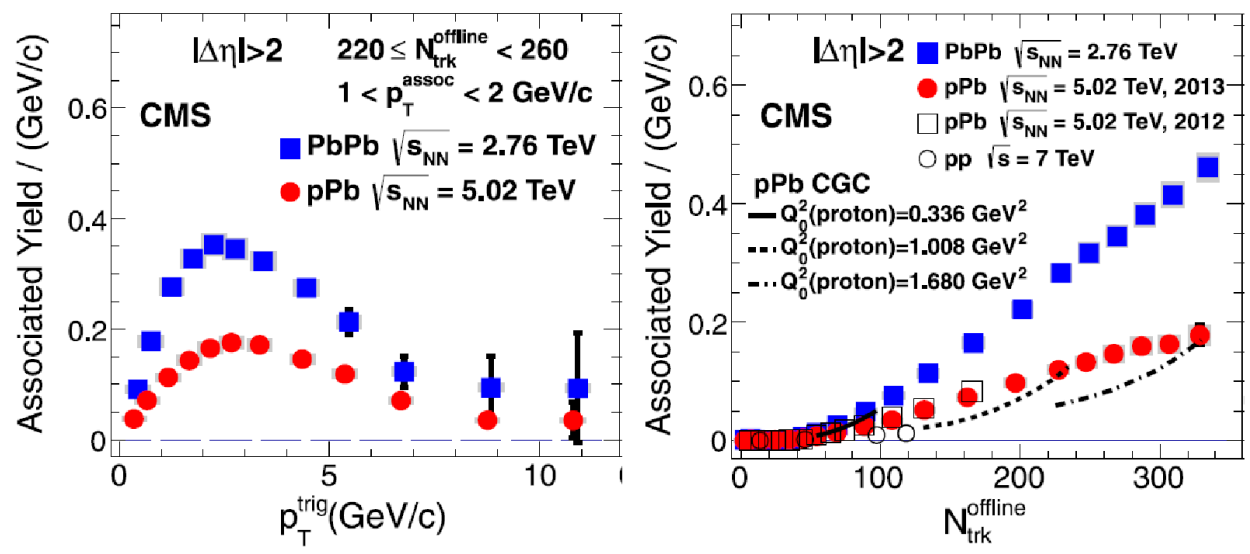

Fig. 2. Event-normalized associated yield for the near-side correlation function integrated over the $|\Delta \phi|<1.2$ region and averaged over the long-range $(|\Delta \eta|>2)$ region [10]. Left panel: Results as a function of $p_{\mathrm{T}}^{\text {trig }}$ for $1<p_{\mathrm{T}}^{\text {assoc }}<2 \mathrm{GeV} / c$ and for events with $220 \leq N_{\text {trk }}^{\text {offline }}<260$. Right panel: Results as a function of multiplicity $N_{\text {trk }}^{\text {offline }}$ for $1<p_{\mathrm{T}}^{\text {trig }}<2 \mathrm{GeV} / c$ and $1<p_{\mathrm{T}}^{\text {assoc }}<2 \mathrm{GeV} / c$. Associated yields from $\mathrm{PbPb}$ collisions at $2.76 \mathrm{TeV}$ (filled squares) and $p \mathrm{~Pb}$ collisions at $5.02 \mathrm{TeV}$ (filled circles) are presented. In addition, the results for $7 \mathrm{TeV} p p$ (open circles) [8] and $5.02 \mathrm{TeV} p \mathrm{~Pb}$ collisions from 2012 run (open squares) [9] as well as calculations from the Color Glass Condensate (CGC) theory (curves) are shown [12].

These include gluon saturation in the initial state of the collision (Color Glass Condensate) — some predictions are shown in Fig. 2 (right panel) [12] — but also hydrodynamic effects in the high-density systems possibly formed in these collisions at $\mathrm{TeV}$ energies $[13,14]$.

To better understand the two-particle correlations observed in $p \mathrm{~Pb}$ and $\mathrm{PbPb}$ collisions, a Fourier decomposition of the $1 \mathrm{D} \Delta \phi$-projected correlation functions for the long-range region $(|\Delta \eta|>2)$ has been performed. Of particular importance are the second and third Fourier coefficients, called the elliptic $\left(v_{2}\right)$ and triangular $\left(v_{3}\right)$ flow, respectively. The coefficients reflect the created medium response to the initial collision geometry and its fluctuations, and allow to study transport properties of the medium using hydrodynamic models. A direct comparison of the extracted elliptic, $v_{2}\{2,|\Delta \eta|>2\}$, and triangular, $v_{3}\{2,|\Delta \eta|>2\}$, flow harmonics for $p \mathrm{~Pb}$ and $\mathrm{PbPb}$ collisions is shown in Fig. 3. The elliptic ${ }^{4}$ and triangular flow increase with multiplicity $\left(N_{\text {trk }}^{\text {offline }}\right)$ in both studied systems. Comparing the $p \mathrm{~Pb}$ and $\mathrm{PbPb}$ data at the same multiplicity, the $v_{2}$ signal exhibits larger magnitude in $\mathrm{PbPb}$ than in $p \mathrm{~Pb}$ collisions, while the $v_{3}$ signal has a remark-

\footnotetext{
${ }^{4}$ The elliptic flow was also obtained using the four-particle cumulant method $-v_{2}\{4\}$.
} 
ably similar magnitude in both systems. This similarity of the triangular flows is not trivially expected since the initial-state collision geometry is very different for the $p \mathrm{~Pb}$ and $\mathrm{PbPb}$ systems.
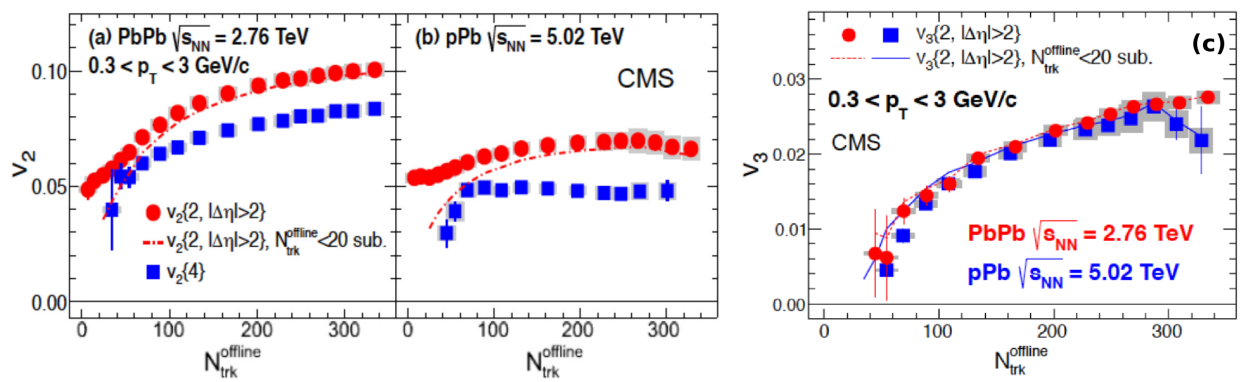

Fig. 3. Left panel: $v_{2}\{2,|\Delta \eta|>2\}$ (filled circles) and $v_{2}\{4\}$ (filled squares) values as a function of $N_{\mathrm{trk}}^{\text {offline }}$ for $0.3<p_{\mathrm{T}}<3 \mathrm{GeV} / c$, in $2.76 \mathrm{TeV} \mathrm{PbPb}$ collisions (a) and $5.02 \mathrm{TeV} p \mathrm{~Pb}$ collisions (b). Right panel (c): $v_{3}\{2,|\Delta \eta|>2\}$ values as a function of $N_{\mathrm{trk}}^{\text {offline }}$ for $0.3<p_{\mathrm{T}}<3 \mathrm{GeV} / c$, in $2.76 \mathrm{TeV} \mathrm{PbPb}$ collisions (filled circles) and $5.02 \mathrm{TeV} \mathrm{pPb}$ collisions (filled squares). Results after subtracting the low-multiplicity data $\left(N_{\text {trk }}^{\text {offline }}<20\right)$ are also shown (curves).

Results for the long-range two-particle correlations might suggest that hydrodynamics could play a role already in high-multiplicity (central) $p \mathrm{~Pb}$ collisions. Has there been other evidence of hydrodynamic-like behaviour in proton-lead collisions?

\subsubsection{Transverse momentum spectra of identified hadrons}

Among the most basic physics observables studied in high-energy heavyion collisions are transverse momentum $\left(p_{\mathrm{T}}\right)$ spectra of produced particles. In such collisions, final-state effects modify the particle momentum spectra. Within the hydrodynamical picture, a transverse expansion of the system (a fireball) formed in the heavy-ion collision results in a mass ordering of the particle transverse momenta. As particles inside the fireball move with a collective velocity, the particles having larger mass will acquire larger momenta. Indeed, the so-called "radial" flow has been observed in high-energy heavy-ion collisions, e.g. in $\mathrm{PbPb}$ collisions at the LHC [15].

Transverse momentum spectra of identified hadrons in $p \mathrm{~Pb}$ collisions at $\sqrt{s_{N N}}=5.02 \mathrm{TeV}$ have been measured by the CMS experiment [16]. Charged pions, kaons, and (anti)protons in the laboratory rapidity ${ }^{5}$ range $|y|<1$ were identified via their energy loss in the silicon tracker. The $p_{\mathrm{T}}$

\footnotetext{
${ }^{5}$ Rapidity $y=\frac{1}{2} \ln \left(\frac{E+p_{z}}{E-p_{z}}\right)$, where $E$ and $p_{z}$ are the energy and longitudinal momentum, respectively.
} 
distributions, obtained for different multiplicity classes ${ }^{6}$ of $p \mathrm{~Pb}$ collisions, are shown in Fig. 4. The particle mean $p_{\mathrm{T}}$ increases with the particle mass. The distributions get also broader for more central collisions, i.e. for larger $\left\langle N_{\text {tracks }}\right\rangle$ values. This behaviour is more directly visible in Fig. 5 (left panel), where the average particle transverse momenta are shown as a function of $\left\langle N_{\text {tracks }}\right\rangle$.
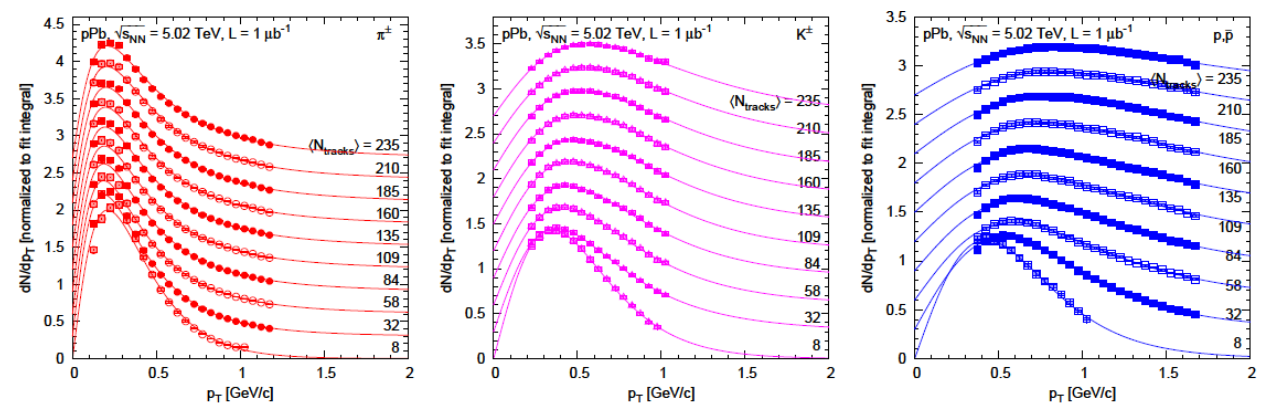

Fig. 4. Transverse momentum distributions of charged pions, kaons, and (anti)protons, in the $|y|<1$ range, for different multiplicity $p \mathrm{~Pb}$ collisions at $\sqrt{s_{N N}}=5.02 \mathrm{TeV}[16]$. Solid lines are fits with the Tsallis-Pareto parametrization. The distributions are normalized such that the fit integral is unity. For better visibility, the result for a given $\left\langle N_{\text {tracks }}\right\rangle$ multiplicity bin is shifted by 0.3 units with respect to the adjacent bins.

The comparison of $\left\langle p_{\mathrm{T}}\right\rangle$ from $p \mathrm{~Pb}$ collisions to $p p$ and $\mathrm{PbPb}$ measurements is presented in Fig. 5 (left panel) as well. Proton-lead and protonproton collisions behave very similarly for low track multiplicities, but at higher multiplicities the average particle transverse momenta are smaller for $p \mathrm{~Pb}$ than for $p p$ collisions. Both the highest-multiplicity $p p$ and $p \mathrm{~Pb}$ interactions yield larger $\left\langle p_{\mathrm{T}}\right\rangle$ than seen in central $\mathrm{PbPb}$ collisions. While in the $\mathrm{PbPb}$ case, even the most central collisions possibly contain a mix of soft $\left(\right.$ low- $\left.p_{\mathrm{T}}\right)$ and hard (high- $p_{\mathrm{T}}$ ) nucleon-nucleon interactions, for $p p$ or $p \mathrm{~Pb}$ collisions the most violent interactions are selected. The track multiplicity dependence of $\left\langle p_{\mathrm{T}}\right\rangle$ indicates that particle production at LHC energies is strongly correlated with the event particle multiplicity in both $p p$ and $p \mathrm{~Pb}$ interactions.

Motivated by the success of Boltzmann-type distributions in $A A$ collisions, the $p_{\mathrm{T}}$ distributions for $p \mathrm{~Pb}$ data were fitted with a function proportional to $p_{\mathrm{T}} \exp \left(-m_{\mathrm{T}} / T^{\prime}\right)$, where $T^{\prime}$ is the inverse slope parameter. The inverse slope parameter as a function of the hadron mass for various multiplicity classes is presented in Fig. 5 (right panel). The $T^{\prime}$ parameter

\footnotetext{
${ }^{6}$ Multiplicity classes are defined by $\left\langle N_{\text {tracks }}\right\rangle$, which is the average number of charged particles (tracks) in the region $|\eta|<2.4$.
} 

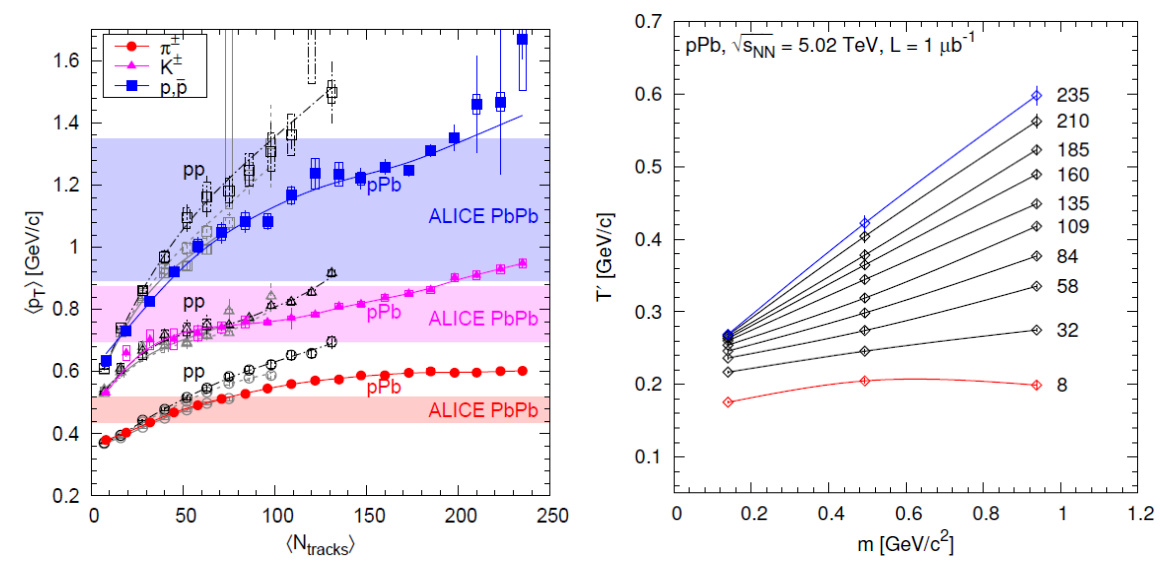

Fig. 5. Left panel: Average transverse momentum of charged pions, kaons and (anti)protons, in the $|y|<1$ range, as a function of multiplicity $\left(\left\langle N_{\text {tracks }}\right\rangle\right)$. Results for $p \mathrm{~Pb}$ collisions at $\sqrt{s_{N N}}=5.02 \mathrm{TeV}$ (filled symbols) [16] are compared to $p p$ collisions at several energies (open symbols) [17]. The ranges of $\left\langle p_{\mathrm{T}}\right\rangle$ measured in different centrality $\mathrm{PbPb}$ collisions at $\sqrt{s_{N N}}=2.76 \mathrm{TeV}$ are also indicated as horizontal bands [15]. Right panel: Inverse slope parameter $T^{\prime}$ of pions, kaons and (anti)protons for different multiplicity classes of $p \mathrm{~Pb}$ collisions [16].

rises with mass and track multiplicity. This may be a hint of the collective (hydrodynamic-like) behaviour in $p \mathrm{~Pb}$ collisions at $\sqrt{s_{N N}}=5.02 \mathrm{TeV}$, since for nucleus-nucleus collisions similar observations [15] are interpreted as due to the radial flow velocity boost.

\subsection{Hard probes}

Given the high centre-of-mass energies achieved in heavy-ion collisions at the LHC, processes that involve a large momentum transfer become abundant. Therefore, the so-called "hard probes" (e.g. high-energy jets, electroweak gauge bosons and high-mass quarkonia) produced in such interactions can be experimentally measured and used to understand properties of the created strongly-interacting medium.

One of the signatures for the formation of QGP is the jet-quenching effect [18]. It has been predicted that interactions of highly energetic partons with the hot and dense coloured matter would lead to the depletion of jet yields at high transverse momenta. First measurements of the parton energy loss were performed at the RHIC accelerator, by studying yields and correlations of high- $p_{\mathrm{T}}$ particles that originate from jet fragmentation $[19,20]$. At LHC energies, jet quenching is investigated both for high- $p_{\mathrm{T}}$ particles and, directly, for fully reconstructed jets. 


\subsubsection{Nuclear modification factors}

A first tool to study jet quenching is the nuclear modification factor, $R_{A A}$, defined as

$$
R_{A A}\left(p_{\mathrm{T}}\right)=\frac{1}{T_{A A}} \frac{d^{2} N^{A A} / d p_{\mathrm{T}} d \eta}{d^{2} \sigma^{N N} / d p_{\mathrm{T}} d \eta}
$$

where $T_{A A}=\left\langle N_{\text {coll }}\right\rangle / \sigma_{\text {inel }}^{N N}$ is the nuclear overlap function. Thus, $R_{A A}$ is the ratio of particle yields in nucleus-nucleus $(A A)$ to nucleon-nucleon $(N N)$ collisions, normalized by $T_{A A}$. The jet-quenching effect is observed when, at high- $p_{\mathrm{T}}, R_{A A}<1$.

The CMS experiment has obtained $R_{A A}$ for various particles as well as jets; the results are shown in Fig. 6. Photons, $Z$ and $W-$ gauge bosons mediating electroweak interactions - play an important role as reference signals for the strongly-interacting probes. In $\mathrm{PbPb}$ collisions at $\sqrt{s_{N N}}=2.76 \mathrm{TeV}$, the normalized yield of isolated (direct) photons [21] as well as yields of $Z \rightarrow \mu^{+} \mu^{-}$[22] and $W \rightarrow \mu \nu$ [23] are consistent with those measured in $p p$ interactions at the same centre-of-mass energy, giving $R_{A A} \approx 1$ (left panel of Fig. 6). For charged hadrons and jets, differently than for the electroweak bosons, nuclear modification factors smaller than unity were measured. In central $\mathrm{PbPb}$ collisions, the high- $p_{\mathrm{T}}$ charged hadrons [24] have $R_{A A} \approx 0.5$, a value very similar to the one found for the inclusive jets [25].
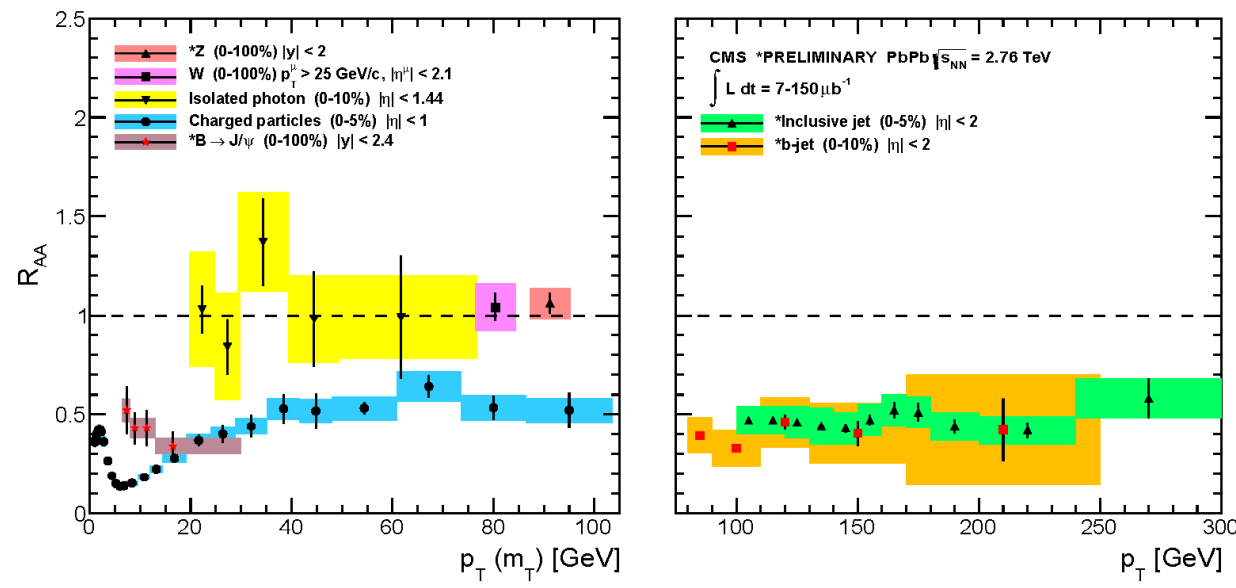

Fig. 6. Left panel: Nuclear modification factors for isolated (direct) photons [21], $Z$ [22] and $W[23]$ bosons, inclusive charged hadrons [24] and $b$-quarks measured by separating secondary $J / \psi$ particles [27]. For $Z$ and $W$, data points are plotted at the rest mass of particles. For charged hadrons, the pion mass is assumed. Centrality selection for each measurement is indicated on the plot. Right panel: Inclusive jet [25] and $b$-jet [28] $R_{A A}$ for central $\mathrm{PbPb}$ collisions as a function of jet $p_{\mathrm{T}}$. 
The energy loss of heavy quarks is expected to be smaller than for light quarks or gluons because of the dead cone effect [26]. To study this flavour dependence of jet quenching, the CMS Collaboration measured in addition $R_{A A}$ for $b$-quarks. The energy loss suffered by $b$-quarks was studied at low transverse momenta by separating non-prompt $J / \psi$ particles (originating from $B$-mesons) from the prompt ones [27], and at high $p_{\mathrm{T}}$ by reconstructing jets in combination with $b$-tagging techniques [28]. This is the first measurement of $b$-jets in heavy-ion collisions. The $b$-quark nuclear modification factor $R_{A A}<1$ (Fig. 6). Contrary to the expectations, the suppression of $b$-jets was found to be comparable to that for inclusive jets, at least within present experimental uncertainties. Thus, $b$-quarks seem to suffer a similar amount of energy loss to light quarks and gluons.

The nuclear modification factor for charged particles produced in $p \mathrm{~Pb}$ collisions at $\sqrt{s_{N N}}=5.02 \mathrm{TeV}$ has also been obtained by CMS [29], and is shown in Fig. 7. As $p p$ collisions at this energy have not been measured, the nuclear modification factor $\left(R_{p \mathrm{~Pb}}\right)$ was obtained by normalizing the measured $p \mathrm{~Pb}$ spectrum to an interpolated $p p$ reference spectrum. The interpolation was done using measured $p p$ data at $0.63,1.8$ and $1.96 \mathrm{TeV}$ collision energies from the $\mathrm{CDF}$ experiment and data at $0.9,2.76$ and $7 \mathrm{TeV}$ collision energies from the CMS experiment. $R_{p \mathrm{~Pb}}$ shows a steady rise to unity at

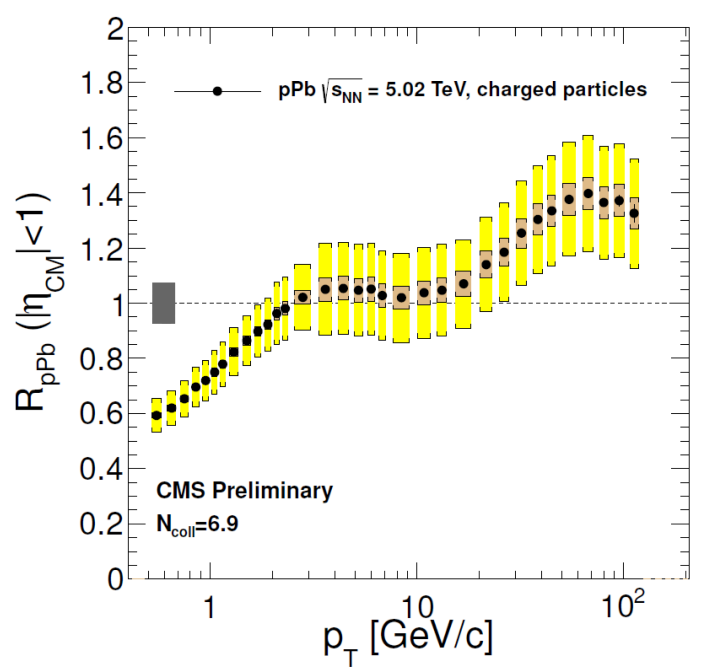

Fig. 7. (Colour on-line) Nuclear modification factor of charged particles from $5.02 \mathrm{TeV} p \mathrm{~Pb}$ collisions [29]. The dark grey band represents the uncertainty of the Glauber calculation of $N_{\text {coll }}$. The grey/light brown band around the measured values shows the uncertainty coming from correlated sources. All other uncertainties are shown by the light grey/yellow band. 
$p_{\mathrm{T}} \approx 3 \mathrm{GeV} / c$, then is flat to approximately $20 \mathrm{GeV} / c$, and at higher $p_{\mathrm{T}}$ it again increases. The rise above unity is in the range of $p_{\mathrm{T}}$ where the parton anti-shadowing is predicted (parton momentum fraction $x=0.02-0.2$ ) [30]. Anti-shadowing may increase the yield of charged particles in $p \mathrm{~Pb}$ collisions compared to the yield in $p p$ collisions.

The measurement of $R_{p \mathrm{~Pb}}$ gives an experimental indication that the jet suppression observed in $\mathrm{PbPb}$ collisions cannot be explained by initial-state effects. Although this result does not point to any jet suppression in $p \mathrm{~Pb}$ collisions, it can suggest the modification of nuclear parton distribution functions (nPDFs) of a Pb-nucleus compared to the free nucleon PDFs. Do we see other signs of initial-state or final-state effects in $p \mathrm{~Pb}$ data?

\subsubsection{Study of dijet events}

Besides nuclear modification factor measurements, the CMS experiment studied jet quenching by analysing also dijet events. In the absence of medium effects, the two jets should have transverse momenta of similar magnitude and be emitted back-to-back. In the case of parton energy losses in the medium, the dijet event characteristics may change, e.g. $p_{\mathrm{T}}$ of jets can be modified.

To characterize the dijet transverse momentum imbalance, an asymmetry ratio was defined as

$$
A_{\mathrm{J}}=\frac{p_{\mathrm{T}, 1}-p_{\mathrm{T}, 2}}{p_{\mathrm{T}, 1}+p_{\mathrm{T}, 2}}
$$

where subscripts 1 and 2 refer to the leading and next-to-leading (subleading) jets in each event. A value of $A_{\mathrm{J}}$ close to zero implies a balanced dijet configuration, and increasing $A_{\mathrm{J}}$ means a stronger suppression of the subleading jet relative to the leading one.

The dijet asymmetry for peripheral and central $2.76 \mathrm{TeV} \mathrm{PbPb}$ collisions is shown in Fig. 8 (upper panel) [31]. $A_{\mathrm{J}}$ was calculated for pairs of jets for which the leading jet $p_{\mathrm{T}, 1}>120 \mathrm{GeV} / c$ and the subleading jet $p_{\mathrm{T}, 2}>50 \mathrm{GeV} / c$ selections were used. In addition, to ensure that dijets are approximately back-to-back, a cut $\Delta \phi_{1,2}=\left|\phi_{1}-\phi_{2}\right|>2 \pi / 3$ was applied. For peripheral $\mathrm{PbPb}$ collisions, the $A_{\mathrm{J}}$ distribution is in good agreement with the reference distribution (PYTHIA+DATA). The dijet momentum imbalance increases with centrality, and for the (0-10\%) most central collisions the imbalance for $\mathrm{PbPb}$ data is significantly larger than that for the reference distribution. This observation is consistent with the jet-quenching scenario. On the other hand, the distribution of the azimuthal angle difference between the two jets, $\Delta \phi_{1,2}$, remains sharply peaked at 180 degrees, as shown in Fig. 8 (bottom panel) [31]. This means that the parton energy losses do not cause a strong angular decorrelation of dijets. 

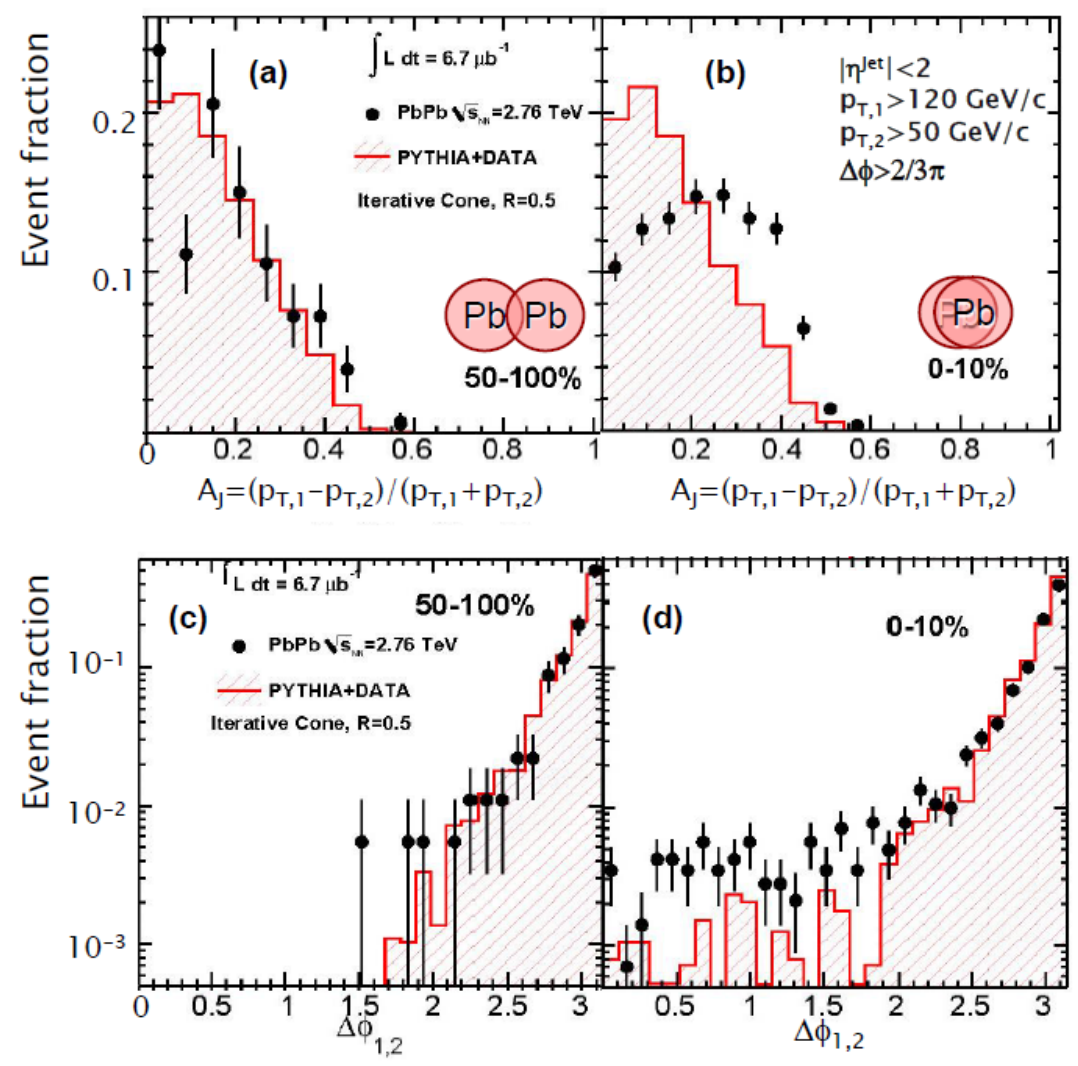

Fig. 8. Upper panel: Dijet asymmetry ratio, $A_{\mathrm{J}}$, for leading jets of $p_{\mathrm{T}, 1}>120 \mathrm{GeV} / c$ and subleading jets of $p_{\mathrm{T}, 2}>50 \mathrm{GeV} / c$, separated in azimuthal angle by $\Delta \phi_{1,2}>$ $2 \pi / 3$, from $2.76 \mathrm{TeV} \mathrm{PbPb}$ data for (a) peripheral $50-100 \%$ and (b) central $0-10 \%$ collisions. Bottom panel: $\Delta \phi_{1,2}$ distributions for (c) peripheral $50-100 \%$ and (d) central $0-10 \% \mathrm{PbPb}$ collisions. Data are shown as points and PYTHIA dijet events embedded into $\mathrm{PbPb}$ data as hatched histograms [31].

A similar study of dijet balance has also been done for $p \mathrm{~Pb}$ collisions at $\sqrt{s_{N N}}=5.02 \mathrm{TeV}$. In order to characterize the dijet transverse momentum balance (or imbalance) quantitatively, the dijet transverse momentum ratio $p_{\mathrm{T}, 2} / p_{\mathrm{T}, 1}$ was used. The dijet transverse momentum balance was studied as a function of the collision centrality, given by the transverse energy measured in the hadronic forward calorimeters $\left(E_{\mathrm{T}}^{\mathrm{HF}[|\eta|>4]}\right)$. In contrast to what is seen in $\mathrm{PbPb}$ collisions, no significant dijet transverse momentum imbalance is observed for $p \mathrm{~Pb}$ collisions with respect to the reference data (PYTHIA and PYTHIA+HIJING), as shown in Fig. 9 (left panel) [32]. Distributions of the dijet azimuthal angle difference $\Delta \phi_{1,2}$ are also in good agreement with the 
simulated reference distributions (see Fig. 9 (right panel)). The dijet results for $p \mathrm{~Pb}$ collisions indicate that jet quenching observed in $\mathrm{PbPb}$ collisions is a final-state effect. Since dijets for $p \mathrm{~Pb}$ data are not significantly modified by final-state effects, a study of initial-state effects for $p \mathrm{~Pb}$ dijet events is possible.
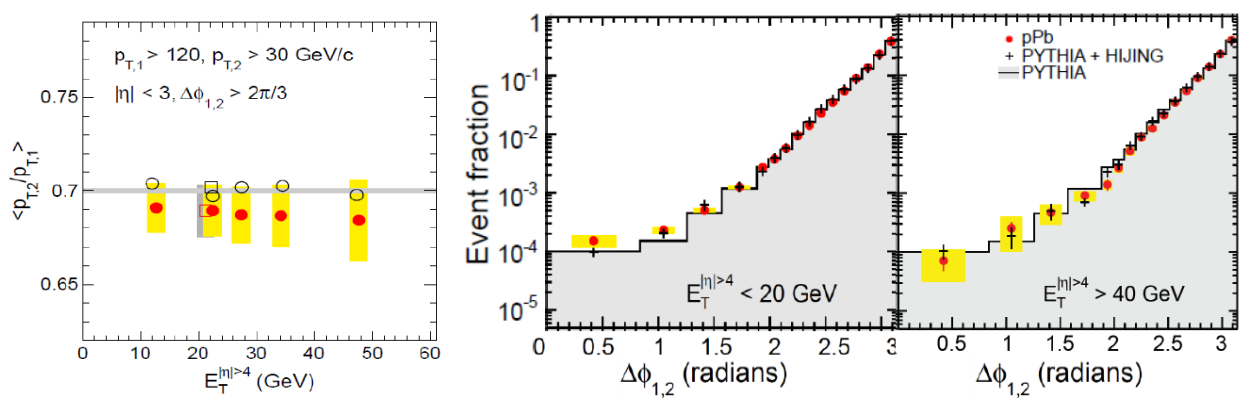

Fig. 9. Left panel: Average ratio of dijet transverse momenta as a function of $E_{\mathrm{T}}^{|\eta|>4}$ for $5.02 \mathrm{TeV} p \mathrm{~Pb}$ data (filled circles), compared to the PYTHIA+HIJING (open circles) and PYTHIA (grey band) simulations. The inclusive results for $p \mathrm{~Pb}$ and PYTHIA+HIJING (empty squares) are also shown [32]. Right panel: Distributions of the azimuthal angle difference $\Delta \phi_{1,2}$ between the leading and subleading jets, for leading jets with $p_{\mathrm{T}, 1}>120 \mathrm{GeV} / c$ and subleading jets with $p_{\mathrm{T}, 2}>30 \mathrm{GeV} / c$, presented for two $E_{\mathrm{T}}^{|\eta|>4}$ classes (peripheral and central collisions). Results for $p \mathrm{~Pb}$ data are shown as filled circles, for PYTHIA+HIJING as crosses and for PYTHIA as a histogram [32].

Dijet production rates as a function of dijet pseudorapidity $\eta_{\text {dijet }}$, defined as $\left(\eta_{1}+\eta_{2}\right) / 2$, were used as a tool to probe the nuclear modifications of the parton distribution functions in $p \mathrm{~Pb}$ collisions. As $\eta_{\text {dijet }}$ and the longitudinal-momentum fraction $x$ of the hard-scattered parton from the $\mathrm{Pb}$-ion are correlated, the $\eta_{\text {dijet }}$ distributions are sensitive to possible modifications of the PDF for nucleons in the lead nucleus when comparing the distributions in $p \mathrm{~Pb}$ and $p p$ collisions.

The $\eta_{\text {dijet }}$ distributions for $p \mathrm{~Pb}$ collisions studied as a function of centrality $\left(E_{\mathrm{T}}^{|\eta|>4}\right.$ classes $)$ are shown in Fig. 10 [32]. The $p \mathrm{~Pb}$ data are compared to PYTHIA and PYTHIA+HIJING simulations, since there is no measured $p p$ reference data at $5.02 \mathrm{TeV}$. A systematic and significant shift of the $\eta_{\text {dijet }}$ distribution is observed as a function of the forward transverse energy. The shift is in the direction of a moving Pb-nucleus (towards negative $\eta_{\text {dijet }}$ values). For each centrality class, deviations of the $\eta_{\text {dijet }}$ distributions with respect to the reference distributions are observed. The modifications of dijet pseudorapidity distributions in $p \mathrm{~Pb}$ collisions are larger than predicted using the NLO calculations based on impact-parameter dependent nPDFs [33]. 


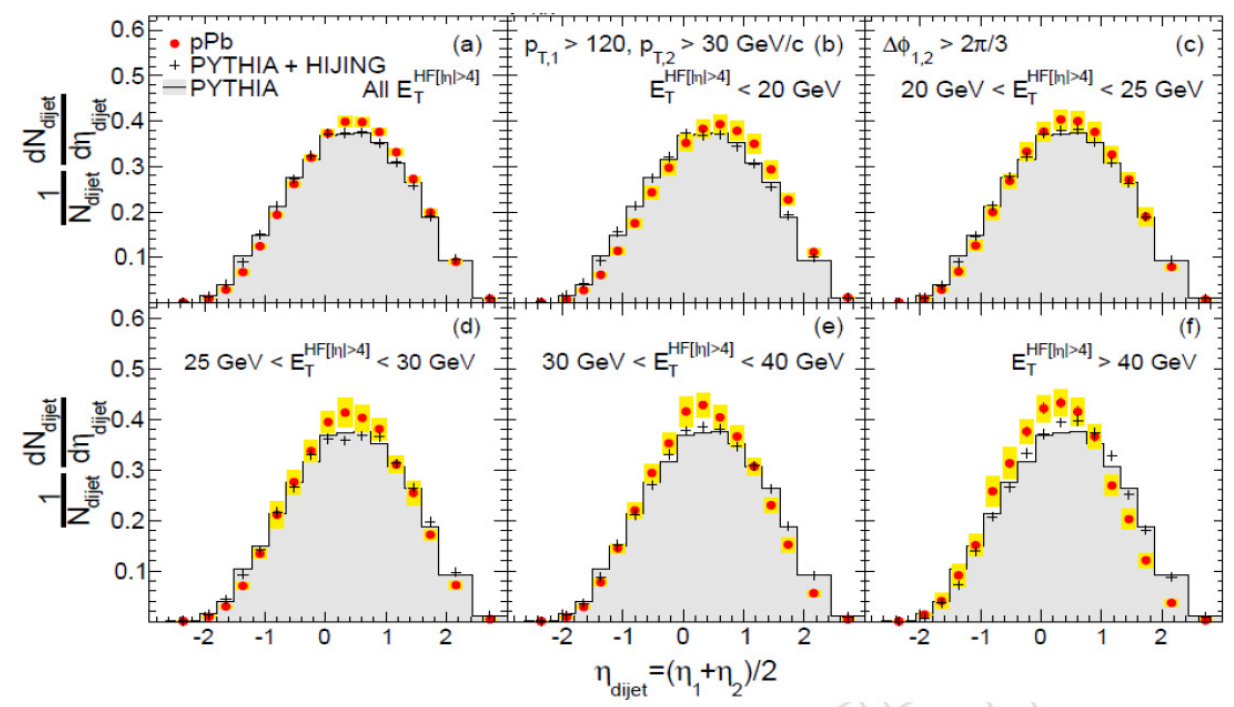

Fig. 10. Dijet pseudorapidity distributions for leading jets with $p_{\mathrm{T}, 1}>120 \mathrm{GeV} / c$ and subleading jets with $p_{\mathrm{T}, 2}>30 \mathrm{GeV} / c$, shown for different $E_{\mathrm{T}}^{|\eta|>4}$ classes. Results for $p \mathrm{~Pb}$ data are shown as filled circles, for PYTHIA+HIJING as crosses and for PYTHIA as a histogram [32].

\subsubsection{Quarkonia suppression}

The suppression of quarkonium states is the next signature of QGP formation in heavy-ion collisions [34]. In QGP, constituents of the quarkonium state, a heavy quark and its antiquark, are screened by the colour charges of the surrounding quarks and gluons. Due to the colour screening, quarkonia would "melt" and become less abundant. Various quarkonium states have different binding energies and radii, therefore, their dissociation temperatures are different [35]. The sequential melting of quarkonium states, depending on their binding energies, is predicted. The states with low binding energies would melt first, and their yields would be suppressed the most.

The excellent resolution of the CMS muon system enabled the measurement of charmonium $(J / \psi, \psi(2 S))$ and bottomonium $(\Upsilon(1 S, 2 S, 3 S))$ families in the dimuon decay channel, for $\mathrm{PbPb}$ and $p p$ collisions at $2.76 \mathrm{TeV}$. Figure 11 shows the nuclear modification factor, $R_{A A}$, for the quarkonia produced in minimum bias $\mathrm{PbPb}$ collisions, as a function of the binding energy. Qualitatively, the sequential melting scenario is confirmed, as the ordering of the yield suppression is consistent with the ordering of binding energies. For quantitative statements, the influence of the $p_{\mathrm{T}}$-cuts and feed-down contributions has to be evaluated. 


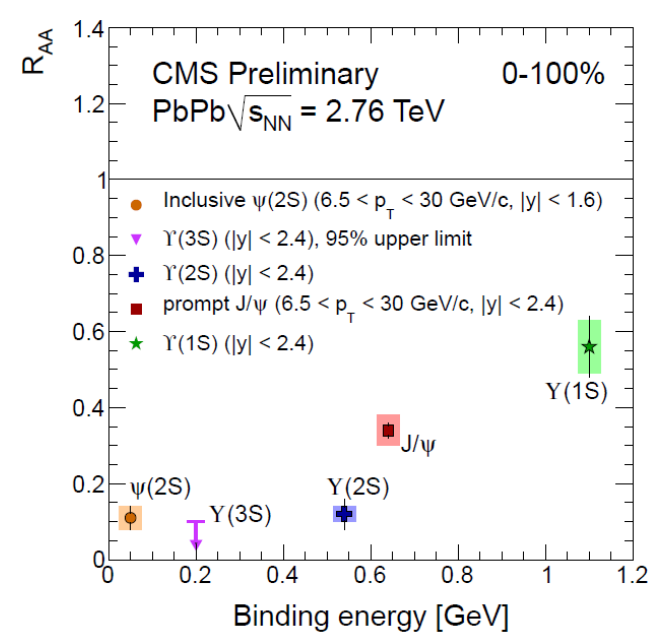

Fig. 11. Minimum bias $R_{A A}$ for all quarkonia measured by CMS: high- $p_{\mathrm{T}}$ states of prompt $J / \psi[27$ ] and inclusive $\psi(2 S)$ [36], as well as $\Upsilon(1 S, 2 S, 3 S)$ states [37]. For $\Upsilon(3 S)$, the upper limit is given.

Competing physics effects, both in the initial and in the final state of the collision, may complicate interpretation of any quarkonia measurement. Further insights in understanding the $\mathrm{PbPb}$ quarkonia results can be gained by studying $p \mathrm{~Pb}$ collisions. Figure 12 shows the results for $\Upsilon$ states measured in $p p$ and $\mathrm{PbPb}$ collisions at the nucleon-nucleon centre-of-mass energy of $2.76 \mathrm{TeV}$ but also in $p \mathrm{~Pb}$ collisions at $\sqrt{s_{N N}}=5.02 \mathrm{TeV}$ [38]. The excited-to-ground-state cross section ratios, $\Upsilon(n S) / \Upsilon(1 S)$, are presented. The centrality-integrated $\Upsilon(2 S) / \Upsilon(1 S)$ and $\Upsilon(3 S) / \Upsilon(1 S)$ ratios are smaller for $p \mathrm{~Pb}$ collisions than for $p p$ collisions but are larger than the corresponding ratios obtained for $\mathrm{PbPb}$ collisions (left panel of Fig. 12). This suggests the presence of final-state suppression effects in $p \mathrm{~Pb}$ collisions compared to $p p$ collisions, which more strongly affect the excited states $(\Upsilon(2 S)$ and $\Upsilon(3 S))$ than the ground state $(\Upsilon(1 S))$. In $\mathrm{PbPb}$ collisions, these effects are even more stronger.

For all the three collision systems, the $\Upsilon(n S) / \Upsilon(1 S)$ ratios are found to decrease with increasing number of charged particles $N_{\text {tracks }}^{|\eta|<2.4}$ (right panel of Fig. 12). The charged particle multiplicity ${ }^{7}$ is measured in the pseudorapidity region containing rapidities of the bottomonium measurements. The $\Upsilon(n S) / \Upsilon(1 S)$ dependence on $N_{\text {tracks }}^{|\eta|<2.4}$ seems to suggest the presence of novel phenomena in quarkonium production. The observed dependence could arise from a larger number of charged particles being systematically

7 The charged particle multiplicity, $N_{\text {tracks }}^{|\eta|<2.4}$, is defined as the number of tracks reconstructed in the tracker, for $|\eta|<2.4$ and $p_{\mathrm{T}}>0.4 \mathrm{GeV} / c$. 
produced with the $\Upsilon(1 S)$ ground state, or from a stronger impact of the growing number of nearby particles on more loosely bound states $(\Upsilon(2 S)$, $\Upsilon(3 S))$.
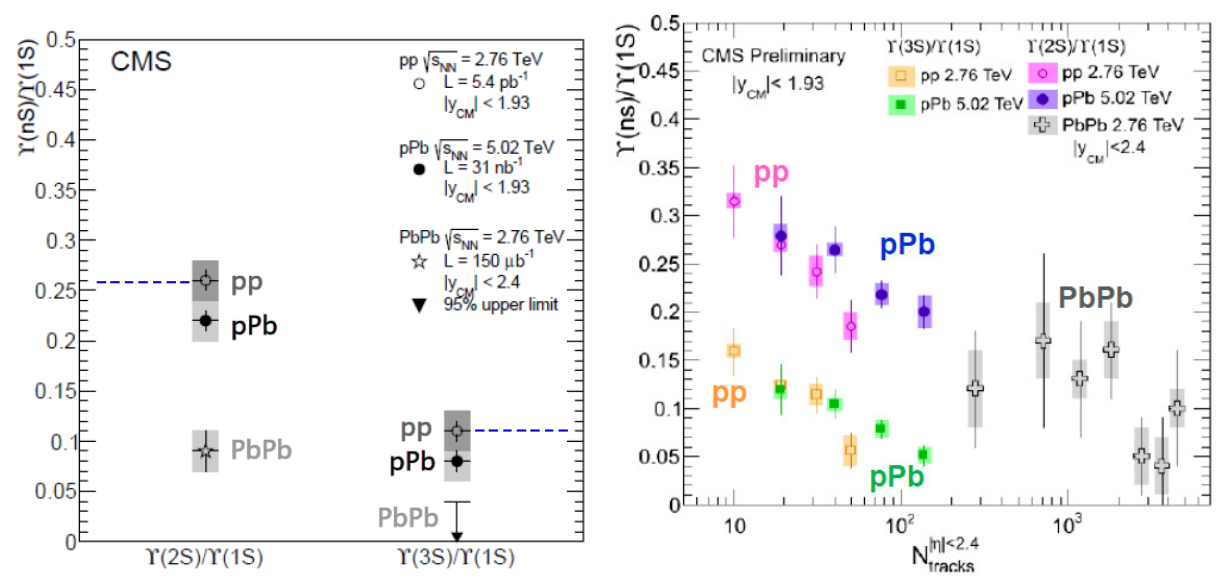

Fig. 12. Left panel: Centrality-integrated single cross section ratios of the excited $\Upsilon(2 S)$ and $\Upsilon(3 S)$ states to the ground $\Upsilon(1 S)$ state, for $p p$ collisions at $2.76 \mathrm{TeV}$ (open circles), $p \mathrm{~Pb}$ at $5.02 \mathrm{TeV}$ (filled circles) and $\mathrm{PbPb}$ at $2.76 \mathrm{TeV}$ (open star) [38]. Right panel: The $\Upsilon(2 S) / \Upsilon(1 S)$ and $\Upsilon(3 S) / \Upsilon(1 S)$ ratios as a function of the number of charged tracks, $N_{\text {tracks }}^{|\eta|<2.4}$, for $p p$ (open circles and squares), $p \mathrm{~Pb}$ (filled circles and squares) and $\mathrm{PbPb}$ (crosses) collisions [38].

\section{Summary}

The CMS heavy-ion programme covers a wide range of physics observables, studied in $\mathrm{PbPb}, p \mathrm{~Pb}$ and $p p$ collisions. The apparatus of CMS enables the investigation of various hard probes but also studies of bulk particle production and collective phenomena. In this paper, the selected results on collective-behaviour phenomena, jet quenching and quarkonia suppression have been presented. Main findings are specified below.

- The analysis of two-particle correlations in $\mathrm{PbPb}, p \mathrm{~Pb}$ and $p p$ collisions at the LHC energies resulted in the observation of the ridge effect in all the three systems. The detailed studies have shown that in $\mathrm{PbPb}$ collisions the effect can be attributed to the hydrodynamic behaviour of the medium. For $p \mathrm{~Pb}$ collisions, the collective phenomena seem to be present as well, as suggest the analyses of the Fourier coefficients $\left(v_{2}\right.$ and $\left.v_{3}\right)$ and charged particle $p_{\mathrm{T}}$ spectra. 
- The nuclear modification factor studies revealed that the medium created in $\mathrm{PbPb}$ collisions does not quench high- $p_{\mathrm{T}}$ photons, $Z$ and $W$ bosons but strongly quenches high- $p_{\mathrm{T}}$ partons (including $b$-quarks). These observations are consistent with jet quenching. The further evidence for the jet-quenching effect comes from the analysis of dijet events. The transverse-momentum imbalance of dijets was studied in detail, pointing to strong parton energy losses in the medium and no significant angular decorrelation of the jets. The measurements performed for $p \mathrm{~Pb}$ collisions indicate that the jet suppression observed in $\mathrm{PbPb}$ collisions cannot be explained by initial-state effects as the phenomenon is not observed in $p \mathrm{~Pb}$ collisions.

- The measurements of $R_{A A}$ for charmonium and bottomonium states confirm the sequential melting of quarkonia in $\mathrm{PbPb}$ collisions. Some quarkonium suppression effects are also present in $p \mathrm{~Pb}$ collisions, as found in the study of the $\Upsilon$ excited-to-ground state ratios.

- Proton-lead collisions are very valuable for the determination of cold nuclear matter effects. For example, the measurements of the nuclear modification factor $\left(R_{p \mathrm{~Pb}}\right)$ and the $\eta_{\text {dijet }}$ pseudorapidity distributions are sensitive to the nuclear parton distribution functions (nPDFs).

\section{REFERENCES}

[1] S. Chatrchyan et al. [CMS Collaboration], JINST 3, S08004 (2008).

[2] M.L. Miller et al., Annu. Rev. Nucl. Part. Sci. 57, 205 (2007).

[3] CMS Collaboration, CMS Public Heavy Ion Results, http://twiki.cern.ch/twiki/bin/view/CMSPublic/PhysicsResultsHIN

[4] B. Abelev et al. [STAR Collaboration], Phys. Rev. C80, 064912 (2009).

[5] B. Alver et al. [PHOBOS Collaboration], Phys. Rev. Lett. 104, 062301 (2010).

[6] S. Chatrchyan et al. [CMS Collaboration], J. High Energy Phys. 1107, 076 (2011).

[7] S. Chatrchyan et al. [CMS Collaboration], Eur. Phys. J. C72, 2012 (2012).

[8] V. Khachatryan et al. [CMS Collaboration], J. High Energy Phys. 1009, 091 (2010).

[9] S. Chatrchyan et al. [CMS Collaboration], Phys. Lett. B718, 795 (2013).

[10] S. Chatrchyan et al. [CMS Collaboration], Phys. Lett. B724, 213 (2013).

[11] B. Abelev et al. [ALICE Collaboration], Phys. Lett. B719, 29 (2013).

[12] K. Dusling, R. Venugopalan, Phys. Rev. D87, 094034 (2013).

[13] P. Bozek, Phys. Rev. C85, 014911 (2012). 
[14] P. Bozek, W. Broniowski, Phys. Lett. B718, 1557 (2013).

[15] B. Abelev et al. [ALICE Collaboration], Phys. Rev. C88, 044910 (2013).

[16] S. Chatrchyan et al. [CMS Collaboration], arXiv:1307.3442 [hep-ex].

[17] S. Chatrchyan et al. [CMS Collaboration], Eur. Phys. J. C72, 2164 (2012).

[18] M. Gyulassy, M. Plumer, Phys. Lett. B243, 432 (1990).

[19] J. Adams et al. [STAR Collaboration], Phys. Rev. Lett. 91, 072304 (2003).

[20] J. Adams et al. [STAR Collaboration], Phys. Rev. Lett. 97, 162301 (2006).

[21] S. Chatrchyan et al. [CMS Collaboration], Phys. Lett. B710, 256 (2012).

[22] CMS Collaboration, Physics Analysis Summary CMS-PAS-HIN-13-004 (2013), http://cds . cern. ch/record/1564677

[23] S. Chatrchyan et al. [CMS Collaboration], Phys. Lett. B715, 66 (2012).

[24] S. Chatrchyan et al. [CMS Collaboration], Eur. Phys. J. C72, 1945 (2012).

[25] CMS Collaboration, Physics Analysis Summary CMS-PAS-HIN-12-004 (2012), http://cds.cern.ch/record/1472722

[26] N. Armesto et al., Phys. Rev. D69, 114003 (2004).

[27] CMS Collaboration, Physics Analysis Summary CMS-PAS-HIN-12-014 (2012), http://cds.cern.ch/record/1472735

[28] S. Chatrchyan et al. [CMS Collaboration], arXiv:1312.4198 [nucl-ex].

[29] CMS Collaboration, Physics Analysis Summary CMS-PAS-HIN-12-017 (2012), http://cms-physics.web.cern.ch/ cms-physics/public/HIN-12-017-pas.pdf

[30] C. Salgado et al., J. Phys. G 39, 015010 (2012).

[31] S. Chatrchyan et al. [CMS Collaboration], Phys. Rev. C84, 024906 (2011).

[32] S. Chatrchyan et al. [CMS Collaboration], arXiv:1401.4433 [nucl-ex].

[33] I. Helenius et al., J. High Energy Phys. 1207, 073 (2012).

[34] T. Matsui, H. Satz, Phys. Lett. B178, 416 (1986).

[35] S. Digal, P. Petreczky, H. Satz, Phys. Rev. D64, 094015 (2001).

[36] CMS Collaboration, Physics Analysis Summary CMS-PAS-HIN-12-007 (2012), http://cds.cern.ch/record/1455477

[37] S. Chatrchyan et al. [CMS Collaboration], Phys. Rev. Lett. 109, 222301 (2012).

[38] S. Chatrchyan et al. [CMS Collaboration], arXiv:1312.6300 [nucl-ex]. 